\title{
Toll of acute methanol poisoning for preventing COVID-19
}

\author{
Omid Mehrpour ${ }^{1,2} \cdot$ Mahmood Sadeghi $^{2}$ (])
}

Received: 24 April 2020 / Accepted: 20 May 2020 / Published online: 27 May 2020

c) Springer-Verlag GmbH Germany, part of Springer Nature 2020

Acute methanol poisoning is a life-threatening type of metabolic and neurological intoxication that commonly occurs following the accidental ingestion of methylated spirits (Kraut and Kurtz 2008). Methyl alcohol toxicity in Iran related to the new coronavirus disease, COVID-19, has attracted the attention of the medical professionals. Just a few days after the widespread coronavirus outbreak, unfounded rumors circulated on social media that drinking or gargling alcohol could prevent or cure COVID-19. Subsequently, hospitals encountered a spike in the referral of poisoning cases for the ingestion of industrial-grade ethanol, resulting in an unprecedented high death toll due to alcohol poisoning. As of April 20, 2020, at the time of writing this letter, 700 people have died as a result of ingesting denatured alcohol in an attempt to fight the novel coronavirus. This catastrophe has affected many cities throughout Iran and at least 3100 people have been hospitalized. The prevalence of COVID-19 has caused a reduction in the amount of ethanol on the market. Tragically, opportunistic individuals have used bleach to mask the color of industrial methanol solutions in order to sell the colorless methyl alcohol instead of drinkable ethanol. As a result, intoxicating liquor was consumed with the false assumption that drinking alcohol would kill the virus, and some of the poisoned patients were intoxicated in this way.

False information and rumors about substances that can supposedly cure or prevent COVID-19 can be just as dangerous and harmful as the virus itself. Nowadays, false information is shared rapidly and widely via social networks. Compare this to the pre-internet era, when data were more likely to be presented based on review and revision by knowledgeable official or professional sources prior to

Mahmood Sadeghi

sadeghim@bums.ac.ir; sadeghi.mahmud@yahoo.com

1 Rocky Mountain Poison and Drug Safety, Denver Health, Denver, CO, USA

2 Medical Toxicology and Drug Abuse Research Center (MTDRC), Birjand University of Medical Sciences, Birjand, Iran dissemination and distribution to the public. In other words, at the present time, misinformation flows readily and confusion is widespread, while in the past, published information was much more likely to have been evaluated by reliable sources and, thus, more trustworthy. Therefore, it is imperative to exert every effort to dispel dangerous and unscientific misinformation. It is of crucial importance that, while fighting the deadly novel coronavirus, we expose misinformation and false rumors in order to protect people and prevent more damage to their health and to the ability of hospitals to function effectively. If we fail to do so, we can expect more people to die from poisoning than from COVID-19. Therefore, it must be emphasized that people should follow recommended medical advice to protect themselves and their families from becoming infected. Finally, it is worth mentioning that ethanol has previously been proven to suppress the immune system; and that COVID-19 has a poor prognosis in anyone who contracts it and has a compromised immune system (Qin et al. 2020). Ethanol can alter innate immunity through functional changes of the innate immune cells. It can also modify the activation of immune cells by specific interactions with cell-membrane molecules (Goral et al. 2008). Therefore, off-label ethanol therapy is not only an unreasonable treatment for COVID-19 but also a highly dangerous one.

Funding None to mention.

\section{Compliance with ethical standards}

Conflict of interest Authors declare that they have no conflict of interest.

\section{References}

Goral J, Karavitis J, Kovacs EJ (2008) Exposure-dependent effects of ethanol on the innate immune system. Alcohol 42(4):237-247 
Kraut JA, Kurtz I (2008) Toxic alcohol ingestions: clinical features, diagnosis, and management. Clin J Am Soc Nephrol 3(1):208-225

Qin C, Zhou L, Hu Z et al (2020) Dysregulation of immune response in patients with COVID-19 in Wuhan, China. Clin Infect Dis. https://doi.org/10.1093/cid/ciaa248 (published online ahead of print, 2020 Mar 12)
Publisher's Note Springer Nature remains neutral with regard to jurisdictional claims in published maps and institutional affiliations. 